\title{
Base de Dados de Propriedades Farmacocinéticas: uma Contribuição Fundamental na Pesquisa de Novos Fármacos
}

\section{Tiago L. Moda e Adriano D. Andricopulo}

Os desafios enfrentados pela indústria farmacêutica são enormes em todas as etapas do processo de descoberta e desenvolvimento de fármacos. Um medicamento para uso em humanos deve apresentar um balanço ideal de eficácia e segurança, bem como de suas propriedades farmacocinéticas. A primeira base de dados brasileira para o estudo de propriedades farmacocinéticas foi lançada recentemente na Universidade de São Paulo, sendo batizada de PK/DB. As informações sobre mais de 1,2 mil fármacos são disponíveis na base $\mathrm{PK} / \mathrm{DB}$, incluindo mais de 2,9 mil medidas de várias propriedades farmacocinéticas. PK/DB possui acesso livre e rápido, incluindo também uma ferramenta integrada de predição in silico de propriedades farmacocinéticas e físicoquímicas. PK/DB é uma contribuição 100\% nacional, representando notável inovação tecnológica na área de $\mathrm{P} \& \mathrm{D}$ de fármacos.

Palavras-chave: propriedades farmacocinéticas; base de dados; fármaco.

The challenges facing the pharmaceutical industry are tremendous at every step of the drug discovery and development process. A drug intended for use in humans should have an ideal balance of efficacy and safety, as well as good pharmacokinetic properties. $\mathrm{PK} / \mathrm{DB}$, a comprehensive, web-based and freely available database incorporates high quality data of drug-like and lead-like molecules for a variety of pharmacokinetic and physicochemical properties, including five models for in silico ADME (Absorption, Distribution, Metabolism, and Excretion) prediction. $\mathrm{PK} / \mathrm{DB}$ is a major Brazilian initiative in the drug discovery area.

Keywords: pharmacokinetic properties; database; drug discovery. 


\section{Introdução}

Aindústria farmacêutica vempassandoporimportantes transformações decorrentes sobretudo dos notáveis avanços científicos e tecnológicos fundamentados em novos paradigmas de natureza intrinsecamente multidisciplinar. A descoberta de novos fármacos de diversas classes terapêuticas tem proporcionado melhorias significativas na qualidade de vida das diversas populações no mundo. ${ }^{1,2} \mathrm{O}$ papel relevante da química medicinal nos processos inovativos da gênese planejada de fármacos é preservado ao longo dos anos nas várias interfaces e conexões entre a química e a biologia. Em particular, os métodos em quimio- e bioinformática têm sido amplamente empregados no processo de pesquisa e desenvolvimento (P\&D) de novos fármacos, encontrando grande aplicação em suas etapas iniciais, incluindo a identificação, seleção e otimização de moléculas bioativas candidatas a novas entidades químicas (NCEs, do inglês, New Chemical Entities). ${ }^{3}$ A integração de métodos computacionais avançados ao trabalho químico e biológico experimental é essencial para a geração de novas moléculas bioativas qualificadas acerca de uma série complexa de propriedades farmacodinâmicas e farmacocinéticas. ${ }^{4,5}$

O processo de descoberta e desenvolvimento de NCEs é longo, complexo e envolve altos investimentos. ${ }^{6}$ Desde a concepção até a introdução de um único fármaco no mercado farmacêutico, são estimados entre 12 e 15 anos em P\&D, com investimentos totais da ordem de US\$ 500-880 milhões, podendo alcançar, em alguns casos, cifras superiores a US\$ 1 bilhão. ${ }^{7-12}$

Embora a indústria farmacêutica ocupe um lugar de destaque nos segmentos mais rentáveis do mercado mundial, o número de novos fármacos lançados no mercado diminuiu sensivelmente na última década. ${ }^{13-16}$ Para alcançar as exigências de produtividade impostas pelos elevados níveis de investimentos em $\mathrm{P} \& \mathrm{D}$ de fármacos, as maiores companhias farmacêuticas do mundo têm adotado como uma de suas estratégias principais o investimento em novas tecnologias para a descoberta de fármacos.

A química combinatória e as triagens biológicas automatizadas em larga escala (HTS, do inglês, HighThroughput Screening) têm proporcionado grandes oportunidades para a descoberta de moléculas bioativas com excelentes propriedades farmacodinâmicas. ${ }^{17,18}$ Entretanto, um dos maiores problemas enfrentados nas fases de pesquisa clínica está associado à falta de propriedades farmacocinéticas apropriadas destas moléculas para o desenvolvimento de um novo medicamento. É importante salientar que a fase farmacodinâmica se refere às interações fármaco-receptor responsáveis pelos efeitos farmacológicos dos fármacos, ao passo que a fase farmacocinética se refere ao caminho que um fármaco faz no organismo, englobando processos de absorção, distribuição, metabolismo e excreção (do acrônimo ADME). A ação terapêutica dos medicamentos somente é possível pela associação efetiva das fases farmacodinâmica e farmacocinética.

No esquema moderno de $\mathrm{P} \& \mathrm{D}$ de fármacos apresentado na Figura 1, os métodos computacionais avançados têm surgido como ferramentas de notável importância no estudo de propriedades farmacocinéticas. Diversos modelos in silico (desenvolvidos em computador), com diferentes níveis de complexidade e capacidade de processamento, têm sido gerados como alternativas úteis frente aos procedimentos experimentais tradicionais (e.g. PAMPA, Сaco-2, MDCK, IAM, HDM). ${ }^{19,20}$ A automação, rapidez e menor custo são algumas das vantagens dos métodos in silico. Estima-se que os investimentos em tecnologias in silico crescerão em torno de $20 \%$ até o ano de 2016 e a farmacocinética faz parte destas estimativas. ${ }^{21,22}$

Embora os estudos de propriedades farmacocinéticas sejam essenciais em $\mathrm{P} \& \mathrm{D}$ de fármacos, os pesquisadores que se dedicam a essas atividades, tanto na academia quanto nas indústrias farmacêuticas, encontram grande dificuldade, pois há pouca disponibilidade de dados padronizados. Com o objetivo de disponibilizar informações farmacocinéticas a comunidade científica brasileira e mundial, a primeira base de dados brasileira para o estudo de propriedades farmacocinéticas, batizada de PK/DB (Database for Pharmacokinetic Properties), foi lançada recentemente por nosso grupo no Laboratório de Química Medicinal e Computacional (LQMC) do Instituto de Física de São Carlos (IFSC) da Universidade de São Paulo (USP). A base PK/DB (http://www. pkdb.ifsc.usp.br) incorpora dados de alta qualidade de 
propriedades farmacocinéticas e físico-químicas de fármacos com elevada diversidade química, pertencendo a várias classes terapêuticas distintas.

\section{Base de Dados PK/DB}

\section{CARACTERIZAÇÃO}

Os dados de propriedades farmacocinéticas e físicoquímicas foram coletados da literatura e de base de dados públicas (http://www.pkdb.ifsc.usp.br/pkdb/literature src.php), resultando em mais de 1,2 mil fármacos e 2,9 mil valores de propriedades. Os compostos da base são identificados por sua estrutura, formula molecular, peso molecular, SMILES, ação farmacológica e propriedades farmacocinéticas. PK/DB também possui um módulo de predição de propriedades de ADME, o qual permite aos usuários submeter seus compostos de interesse para avaliação de propriedades como absorção intestinal humana, biodisponibilidade oral humana, ligação às proteínas plasmáticas, permeabilidade da barreira hemato-encefálica e solubilidade em água.

\section{PROPRIEDADES FARMACOCINÉTICAS DA BASE PK/DB}

Absorção Intestinal Humana. A absorção intestinal humana (HIA, do inglês, Human Intestinal Absorption) é uma propriedade fundamental para que os medicamentos administrados por via oral possam atingir a corrente sangüínea (via sistêmica) dos pacientes em concentrações necessárias para exercer o seu efeito farmacológico. ${ }^{23} \mathrm{Os}$ valores de HIA presentes na base $\mathrm{PK} / \mathrm{DB}$ são referentes à percentagem da dose administrada por via oral que alcança a veia porta (Tabela 1).

Biodisponibilidade Oral Humana. A biodisponibilidade oral (F) é uma das propriedades farmacocinéticas mais importantes dos fármacos administrados por via oral. É expressa como o valor percentual da dose administrada por via oral que atinge a circulação sistêmica e torna-se disponível na periferia do sítio alvo de ação. ${ }^{19} \mathrm{~A}$ indústria farmacêutica tem como foco absoluto o desenvolvimento de NCEs que possam ser administradas por via oral. ${ }^{24} \mathrm{PK} / \mathrm{DB}$ apresenta centenas de valores de biodisponibilidade associados às estruturas químicas dos fármacos correspondentes (Tabela 1).

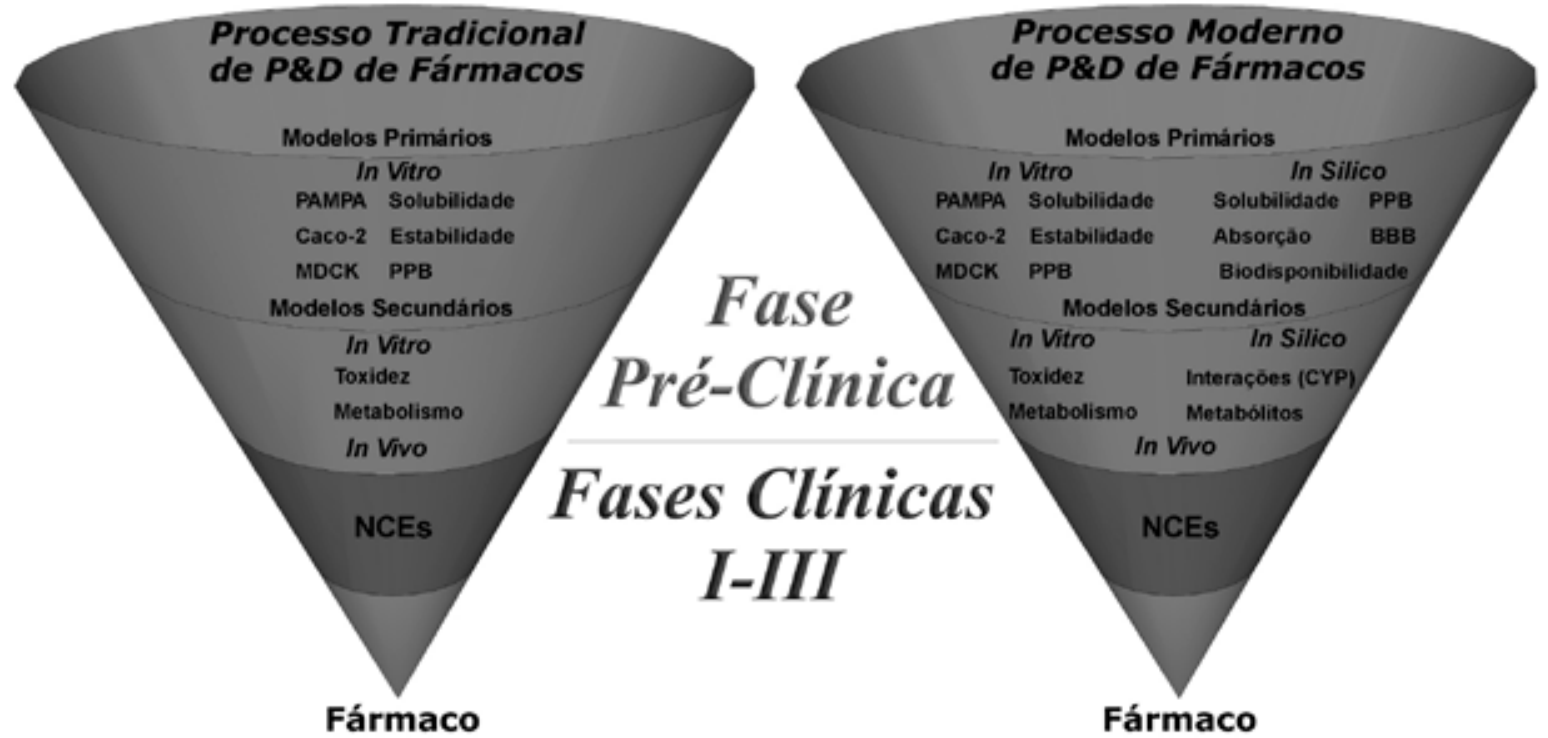

Figura 1: Esquema da transição do processo de pesquisa tradicional para o processo moderno de P\&D de fármacos. A introdução de métodos in silico para o estudo de propriedades farmacocinéticas é uma etapa marcante desta transição. 
Tabela 1: Números de valores de propriedades presentes na base PK/DB

\begin{tabular}{|l|l|l|}
\hline Sigla & Propriedade & Valores \\
\hline HIA & Absorção Intestinal Humana & 677 \\
\hline F & Biodisponibilidade Oral Humana & 660 \\
\hline PPB & Ligação às Proteínas Plasmáticas & 440 \\
\hline BBB & Permeabilidade da Barreira Hemato-Encefálica & 200 \\
\hline Vd & Volume de Distribuição & 291 \\
\hline Cl & Eliminação Renal & 360 \\
\hline T1/2 & Meia-Vida & 355 \\
\hline
\end{tabular}

Ligação às Proteínas Plasmáticas. Após serem absorvidos, os fármacos alcançam a circulação sistêmica e são rapidamente distribuídos em função da alta diferença de concentração entre sítio de absorção, sangue e tecidos. Porém, a distribuição ocorre de forma desuniforme nos diferentes tecidos. A ligação às proteínas plasmáticas (PPP, do inglês, Plasma Protein Binding) exerce um papel central na ação dos medicamentos, afetando diretamente a distribuição dos fármacos livres nos vários tecidos do corpo humano. ${ }^{20}$ Os valores presentes na base PK/DB (Tabela 1) são relacionados à percentagem de fármaco ligado às proteínas do plasma humano.

Barreira Hemato-Encefálica. Outra propriedade importante em $\mathrm{P} \& \mathrm{D}$ de fármacos é a permeabilidade da barreira hemato-encefálica (BBB, do inglês, Blood Brain Barrier), onde células endoteliais firmemente unidas formam uma barreira de transporte para certas substâncias químicas entre os capilares cerebrais e o tecido cerebral. ${ }^{22}$ Essa propriedade tem fundamental importância para os fármacos que atuam no tratamento de doenças do sistema nervoso central (SNC), como epilepsia, mal de Alzheimer, esquizofrenia e tumores cerebrais. Por outro lado, fármacos que não atuam no SNC devem apresentar limitada capacidade de transpor a BBB, minimizando assim, possíveis efeitos adversos. ${ }^{25}$ A Tabela 1 apresenta o número de compostos presentes na base $\mathrm{PK} / \mathrm{DB}$ com valores disponíveis de $\log \mathrm{BB}$ (logaritmo do valor de BBB).

Volume de Distribuição, Eliminação Renal e MeiaVida. O volume de distribuição (Vd), a eliminação renal (Cl) e a meia-vida (T1/2) são importantes propriedades que afetam sensivelmente a ação farmacológica das substâncias bioativas e estão diretamente relacionadas com forma de administração dos medicamentos. ${ }^{21} \mathrm{Vd}$ é usado para quantificar a distribuição do fármaco no corpo humano. PK/DB possui 291 valores de Vd (Tabela 1). $\mathrm{A} \mathrm{Cl}$ é expressa como a percentagem de fármaco absorvido que é eliminada através dos rins e excretada pela urina. PK/DB contém diversos valores de $\mathrm{Cl}$ associados a diferentes estruturas químicas (Tabela 1). A meia-vida (T1/2) se refere ao tempo necessário para que a concentração plasmática de determinado fármaco seja reduzida pela metade. PK/DB apresenta mais de 300 valores de T1/2 (Tabela 1 ).

\section{ESTRUTURA E APLICAÇÕES}

PK/DB permite a busca de compostos por estrutura química, subestrutura, nome e formula molecular, bem como a busca por valor da propriedade farmacocinética especifica ou por faixas de valores de propriedades. A informação sobre a isoforma CYP responsável pelo metabolismo de diversos fármacos também é disponível. Os usuários podem ainda empregar uma combinação de diversos critérios (http:// www.pkdb.ifsc.usp.br/manual. pdf) na busca de informações especificas. Para facilitar a análise, os resultados são mostrados em duas fases distintas. Na primeira fase, o usuário pode escolher o número de compostos a ser exibido no cabeçalho da seção Search e o resultado inicial pode ser organizado de acordo com vários parâmetros da base. A coluna de resultados da primeira fase apresenta a identificação $\mathrm{PK} / \mathrm{DB}$ (MID), estrutura 2D, nome padrão dos 
compostos, SMILES, peso molecular e propriedades farmacocinéticas como mostrado na Figura 2. A segunda fase possibilita ao usuário, através de uma ligação (do inglês, link) direta no nome dos compostos, acesso a informações detalhadas da base como estrutura 3D, propriedades farmacocinéticas, propriedades físicoquímicas e ação farmacológica.

\section{PREDIÇÃO DE PROPRIEDADES FARMACOCINÉTICAS}

A base PK/DB integra cinco modelos in silico para a avaliação de propriedades farmacocinéticas e físicoquímicas, incluindo biodisponibilidade oral humana, ligação às proteínas plasmáticas, absorção intestinal humana, permeabilidade da barreira hemato-encefálica e solubilidade em água. Os modelos apresentam elevada consistência interna e externa e foram desenvolvidos em nossos laboratórios empregando uma técnica de fragmentos moleculares especializados..$^{19,20}$ Pesquisadores do Brasil e de todo mundo podem testar suas moléculas em fase de desenvolvimento nos modelos da base PK/DB.

\section{Conclusões}

As propriedades farmacocinéticas têm recebido grande atenção da indústria farmacêutica mundial, especialmente nas duas últimas décadas. Devido à importância dos processos de ADME durante os estágios iniciais de $\mathrm{P} \& \mathrm{D}$ de fármacos, avanços significativos vêm sendo realizados no desenvolvimento de novas metodologias nos campos in vitro, in vivo e in silico. A base de dados PK/DB representa notável inovação tecnológica, uma contribuição de grande importância para a ciência brasileira na área de $\mathrm{P} \& \mathrm{D}$ de fármacos. $\mathrm{PD} / \mathrm{DB}$ é uma base de dados de acesso livre, contando com uma série de funcionalidades que podem ser acessadas em tempo real, de forma simples, rápida e eficaz, basta que o usuário tenha um computador comum e acesso a internet.

(a)
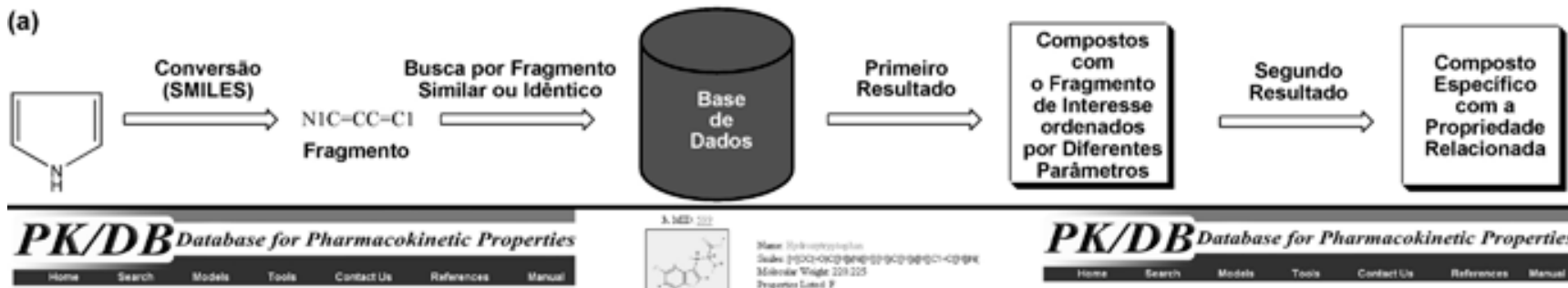

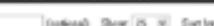
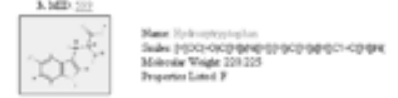

$P K / D B$ Database for Pharmacokinetic Properties

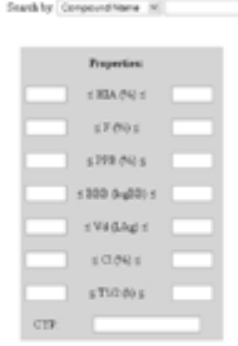

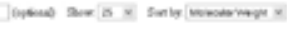
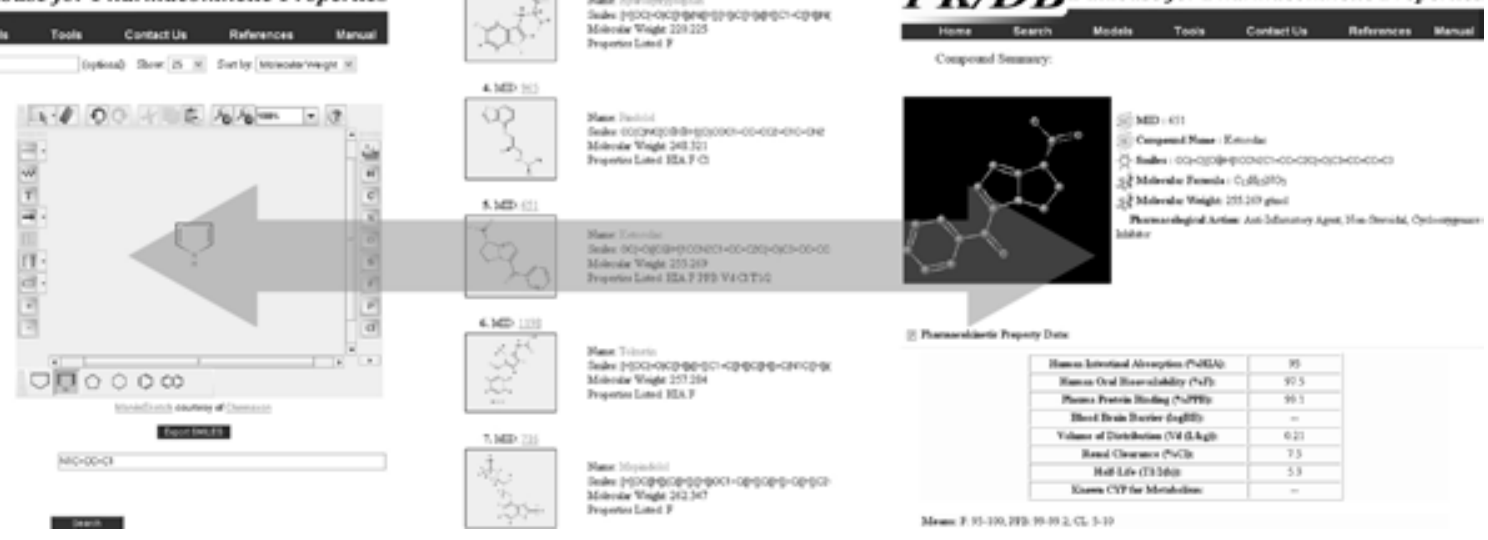

(b)

Figura 2: PK/DB é a primeira base de dados de propriedades farmacocinéticas brasileira. (a) Uma visão geral da arquitetura do sistema da base PK/ DB. (b) Esquema ilustrativo das etapas de busca e análise de resultados. 


\section{REFERÊNCIAS}

1. Caprino, L.; Russo. P.; Drug Discov. Today 2006, 11, 999 .

2. Yago, G.; Amram, M.; Magula, T.; Drug Discov. Dev. 2006, 12, 22.

3. Guido, R. V. C.; Oliva, G.; Andricopulo, A. D.; Curr. Med. Chem. 2008, 15, 37.

4. Jónsdóttir, S. Ó.; Jorgensen, F. S.; Brunak, S.; Bioinformatics 2005, 21, 2145.

5. Andricopulo, A. D.; Montanari, C. A.; Mini Rev. Med. Chem. 2005, 5, 585.

6. Berndt, E. R.; Gottschalk, A. H. B.; Philipson, T. J.; Strobeck, M. W.; Nat. Rev. Drug Discov. 2005, 4, 545.

7. DiMasi, J. A.; Hansen, R. W.; Grabowski, H. G.; J. Health Econ. 2003, 22, 151.

8. Lombardino, J. G.; Lowe III, J. A.; Nat. Rev. Drug Discov. 2004, 3, 853.

9. Boston Consulting Group. A revolution in R\&D: how genomics and genetics are transforming the biopharmaceutical industry. (Boston, Massachusetts, 2001, http://www.bcg.com/publications/files/eng genomicsgenetics_rep_11_01.pdf)

10. Netterwald, J.; Drug. Discov. Dev. 2006, 12, 17.

11. Couzin, J.; Science 2005, 309, 728.

12. Fee, R.; Drug Discov. Dev. 2007, 3, 32.

13. Avorn, J.; Science 2005, 309, 669.

14. McGee, P.; Drug Discov. Dev. 2006, 9, 16.

15. Hoag, H.; Nature 2006, 440, 1084.
16. Mervis, J.; Science 2005, 309, 721.

17. Emilsson, V.; Nature 2008, 452, 423.

18. Shaffer, C.; Drug Discov. Dev. 2008, 5, 36.

19. Moda, T. L.; Montanari, C. A.; Andricopulo, A. D.; Bioorg. Med. Chem. 2007, 15, 7738.

20. Moda, T. L.; Montanari, C. A.; Andricopulo, A. D.; Lett. Drug. Des. Discov. 2007, 4, 502.

21. van de Waterbeemd, H.; Gifford, E.; Nat. Rev. Drug Discov. 2003, 2, 192.

22. Cecchelli, R.; Berezowski, V.; Lundquist, S.; Culot, M.; Renftel, M.; Dehouck, M. P.; Fenart, L.; Nat. Rev. Drug Discov. 2007, 6, 650.

23. Linnankoski, J.; Ranta, V. P.; Yliperttula, M.; Urtt, A.; Eur. J. Pharm. Sci. 2008, 34, 129.

24. Leeson, P. D.; Springthorpe, B.; Nat. Rev. Drug Discov. 2007, 6, 881.

25. Bryan, J.; Pharm. J. 2004, 273, 475.

\section{Tiago L. Moda' e Adriano D. Andricopulo*1}

${ }^{1}$ Laboratório de Química Medicinal e Computacional. Grupo de Cristalografia. Instituto de Física de São Carlos - IFSC/USP, Caixa Postal 369 - Av. Trabalhador São Carlense, 400, CEP: 13560-590 - São Carlos/SP

*E-mail: aandrico@ifsc.usp.br 